\title{
Improvement of the Microwave Properties of Y-Ba-Cu-O Films with Artificial Defects
}

\author{
Roger Wördenweber, Peter Lahl, and Jan Einfeld
}

\begin{abstract}
In this paper, the potential of defects for optimizing the microwave properties of $\mathrm{YBa}_{2} \mathrm{Cu}_{3} \mathrm{O}_{7}$ (YBCO) thin films is demonstrated. On one hand, microscopic $\mathrm{Y}_{2} \mathrm{O}_{3}$ precipitates, which can be created in YBCO thin films by modification of the deposition process, serve as ideal scattering centres for quasiparticles and, thus, lead to a considerable reduction of the microwave surface resistance $R_{s}$. The modification $R_{s}(T)$ can be explained in terms of the two-fluid model. Data for the quasiparticle scattering rate can be obtained from the measurements. On the other hand, the impact of artificial defects, so called antidots, upon the microwave properties is analyzed. $R_{s}$ measurements demonstrate that the ion beam etching creates a $-20 \mathrm{~nm}$ broad damaged area at the edge of the antidots. First measurements of the power handling capability of YBCO thin film resonators indicate that the magnetic contribution to the nonlinear behavior can be reduced by antidots. The implementation of antidots, which have been proven to be an ideal and easy tool to improve active $\mathrm{YBCO}$ thin film devices, might be of use for microwave applications as well.
\end{abstract}

Index Terms-superconductor, high temperature superconductor, thin film, microwaves

\section{INTRODUCTION}

T he use of superconducting films in microwave devices in principle allows a dramatic reduction of the device dimension at comparable or higher performance with respect to devices fabricated of conventional materials. As a consequence, larger power densities are encountered in these devices. However, due to the complex surface impedance $\mathrm{Z}_{\mathrm{s}}=\mathrm{R}_{\mathrm{s}}+\mathrm{iX}$ (surface resistance $\mathrm{R}_{\mathrm{s}}$ and surface reactance $\mathrm{X}_{\mathrm{s}}$ ) nonlinearities in $R_{s}$ are observed, which present a serious limitation for the rf application of these materials. Therefore, it is important to minimise $R_{s}$ and to improve the power handling capability.

In this paper we demonstrate that $\mathrm{R}_{\mathrm{s}}$ and power handling capability of $\mathrm{YBa}_{2} \mathrm{Cu}_{3} \mathrm{O}_{7}$ (YBCO) films can be improved significantly by defects in the film. First, a reduction of $R_{s}$ is obtained by the presence of adequate defects, i.e. $\mathrm{Y}_{2} \mathrm{O}_{3}$ precipitates, which are generated by ion bombardment during sputter deposition. The reduction of $R_{s}$ with increasing density of defects is explained in terms of the two-fluid model with thermally excited quasiparticles characterised by a Drude-shaped conductivity spectrum. Second, first experiments are given which motivate a potential

Manuscript received July 19,2000. This work was supported in part by the DFG Grant No. WO549/3-1 and ESF scientific program VORTEX.

P. Lahl, J. Einfeld and R. Wördenweber are with the Institut für Schichtund Ionentechnik, Forschungszentrum Jülich, 52425 Jülich, Germany (telephone: 49-2461-61-2365, e-mail: r.woerdenweber@fz-juelich.de). improvement of the power handling capability due to artificial defects. In this case micrometer holes (antidots) are patterned into superconducting coplanar stripline resonators. The modification of $R_{s}$ due to the antidots is explained and the shift of the nonlinearity to larger rf power is measured.

\section{MiCROWAVE SURfACE RESISTANCE}

YBCO films are prepared by on-axis dc magnetron sputtering on $r$-cut sapphire and $\mathrm{LaAlO}_{3}$ substrates which have been in-situ buffered with rf-sputtered (001) $\mathrm{CeO}_{2}$ of various thicknesses. One of the advantages of the highpressure magnetron sputtering technique is given by the possibility to create microscopic structural defects in the YBCO film via intentional ion bombardment of the film during the growth [1]. The density and size of the defects can be adjusted by modification of the ion energy which can be controlled by the bias voltage of the cathode. Fig. 1 presents the surface morphology and structural properties of two YBCO films which are prepared under the same conditions except for the ion energy. With increasing ion energy the density of outgrowths, pores and $\mathrm{Y}_{2} \mathrm{O}_{3}$ precipitates increases.

Fig. 2 represents the comparison of the temperature dependence of $R_{s}$ for YBCO films on sapphire and $\mathrm{LaAlO}_{3}$ substrates with different defect densities. Different $R_{s}$ vs. $T$ characteristics are obtained, which seem to depend on the density of defects and clearly not on the substrate material or lattice mismatch between the substrate material and YBCO. For both substrates $\left(\mathrm{Al}_{2} \mathrm{O}_{3}\right.$ and $\left.\mathrm{LaAlO}_{3}\right)$ the YBCO films with low defect density reveal a shoulder or plateau at a temperature range of $(0.5-0.6) \mathrm{T}_{c}$, whereas this shoulder is absent for YBCO films with enhanced defect concentration. Furthermore, smaller values of $\mathrm{R}_{\mathrm{s}}$ are encountered at the technically relevant temperature range $\mathrm{T}<(0.7-0.8) \mathrm{T}_{\mathrm{c}}$ for the


Fig. 1. TEM and SEM images of two YBCO film on $\mathrm{CeO}_{2}$ buffered sapphire which are sputtered using identical standard sputter parameters but slightly different cathode potentials of $280 \mathrm{~V}$ (left) and $284 \mathrm{~V}$ (right). 


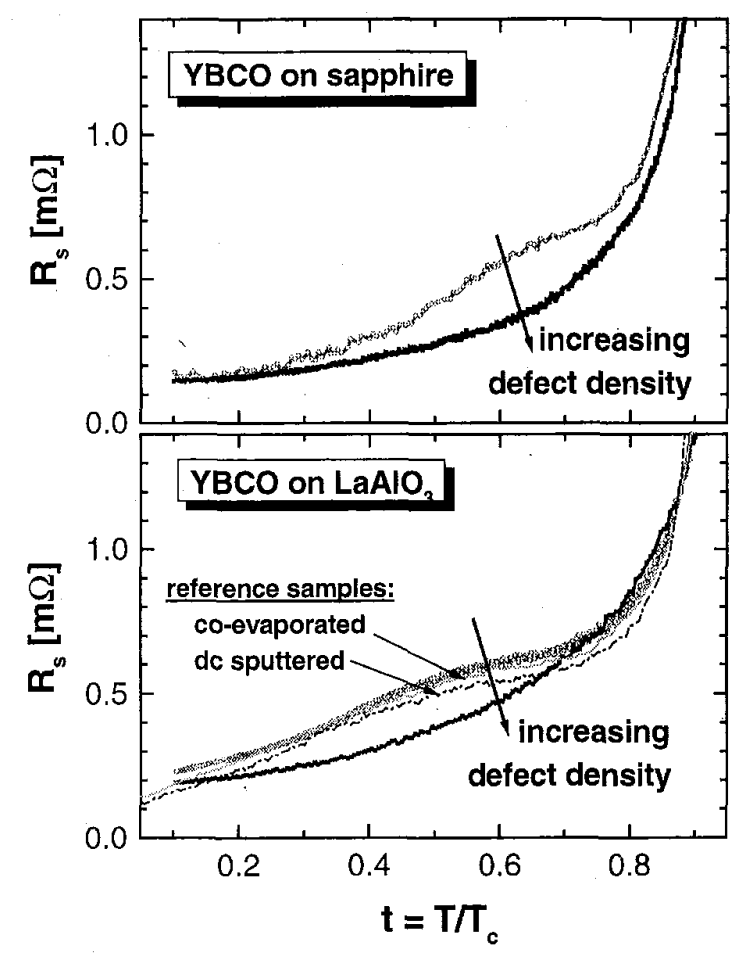

Fig. 2. Temperature dependence of the surface resistance of YBCO films prepared at different ion energies on sapphire (a) and $\mathrm{LaAlO}_{3}$ (b). For comparison, literature data [2] for YBCO films on $\mathrm{LaAlO}_{3}$ prepared by different deposition techniques are shown.

films with high defect density. It should be noted that our YBCO films on $\mathrm{LaAlO}_{3}$ with high defect density have not been optimised up to now. This pronounced difference in the temperature dependence of $R_{s}$ as well as the reduction of $R_{s}$ can be described in terms of the two-fluid model in combination with a large density of microscopic defects [1]. For temperatures not too close to $\mathrm{T}_{\mathrm{c}}$ one obtains:

$$
R_{s}(\omega, T) \cong 0.5 \mu_{o}^{2} \omega^{2} \lambda^{3}(\omega, T) \sigma_{1}(\omega, T)
$$

with the real part of the complex conductivity

$$
\sigma_{1}(\omega, T)=\frac{n_{n}(T) e^{2} \tau(T)}{m \cdot\left(1+\omega^{2} \tau^{2}(T)\right)}
$$

defined by the frequency $\omega$, the quasi-particle density $\mathrm{n}_{n} \propto\left(\mathrm{T} / \mathrm{T}_{\mathrm{c}}\right)^{\beta}$ with $\beta \approx 2$ for HTS material $[1,3]$, and the quasiparticle scattering time $\tau$, which according to Matthiesen rule consists of the contributions due to scattering at impurities, phonons and electrons

$$
\tau^{-1}=\tau_{i m p}^{-1}+\tau_{c l-p h}^{-1}+\tau_{c l-e l}^{-1} \approx \tau_{i m p}^{-1}+\tau_{b}^{-1}\left(T / T_{c}\right)^{\gamma}
$$

with $\gamma \approx 1-2$ for electron-electron dominated scattering and $\gamma \approx 5$ for electron-phonon dominated scattering.

Inserting experimental parameters [1], $\tau$ can be derived from the data using eqs. (1-2). Fig. 3 shows the resulting scattering rate of the thermally excited quasiparticles of two YBCO thin films with different defect densities. For comparison, literature data obtained from YBCO single crystals [4] are added. A number of interesting features can be observed: (i) All experimental curves can be fitted according to eq. (3) using similar parameters $\tau_{b}=(0.075 \pm 0.025) \mathrm{ps}$ and $\gamma=5 \pm 0.4$ [1]. This indicates, that one common mechanism seems to be responsible for the scattering in $\mathrm{YBCO}$ independent of sample and crystalline quality.

(ii) The fit of the experimental data in a double logarithmic plot yields a slope $\gamma \approx 5$ below $\mathrm{T}_{c}$. This behaviour can be interpreted in terms of two different schemas: (a) Either the electron-electron scattering, which is the dominating mechanism in the normal states, persists to dominate the superconducting state below $\mathrm{T}_{\mathrm{c}}$ and the strong temperature dependence $\tau^{-1} \propto \mathrm{T}^{5}$ is a consequence of the reduction of quasiparticle density, i.e, scattering centers, or (b) due to the reduction of the quasiparticle density, the electron-phonon scattering takes over below $\mathrm{T}_{\mathrm{c}}$ and dominates the temperature dependence. Unfortunately, our measurement can not distinguish between these possible explanations.

(iii) In contrast to the parameters $\tau_{\mathrm{b}}$ and $\gamma$, the impurity or defect induced scattering parameter $\tau_{\text {imp }}$ differs strongly from sample to sample. For the single crystals, values of $\tau_{i m p}=19 \mathrm{ps}$ and $1.9 \mathrm{ps}$ are derived from literature data. The large difference has to be ascribed to differences in sample quality with respect to the density of scattering centres. It originates from different growth processes of the two single crystals [4]. The defect density (and thus the density of scattering centres) is expected to be even larger in epitaxial films compared to that in single crystals. This tendency is confirmed by our results. We derived values for our $\mathrm{YBCO}$ films on $\mathrm{Al}_{2} \mathrm{O}_{3}$ and $\mathrm{LaAlO}_{3}$ ranging from $\tau_{\text {imp }}=1.0 \mathrm{ps}$ (low defect density) to $\tau_{\text {imp }}=0.34 \mathrm{ps}$ (high defect density). Obviously $\tau_{i m p}$, decreases with increasing density of defects. Using the simple relation for the mean free path at zero temperature $l_{\mathrm{T}=0}=v \tau_{i m p}$ with the Fermi velocity $v \approx(1.6-1.7) \cdot 10^{5} \mathrm{~m} / \mathrm{s}$ for YBCO [5] yields values ranging from $l_{T \rightarrow 0 \mathrm{~K}} \approx(0.3-3.0) \mu \mathrm{m}$ (single crystal) to $l_{\mathrm{T} \rightarrow 0 \mathrm{~K}} \approx(56-180) \mathrm{nm}$ for our YBCO films. Although there is no direct relation between the defect density and the mean free path ( $l$ depends on the kind and density of defects) these numbers provide a qualitative description of the morphology of the different samples.

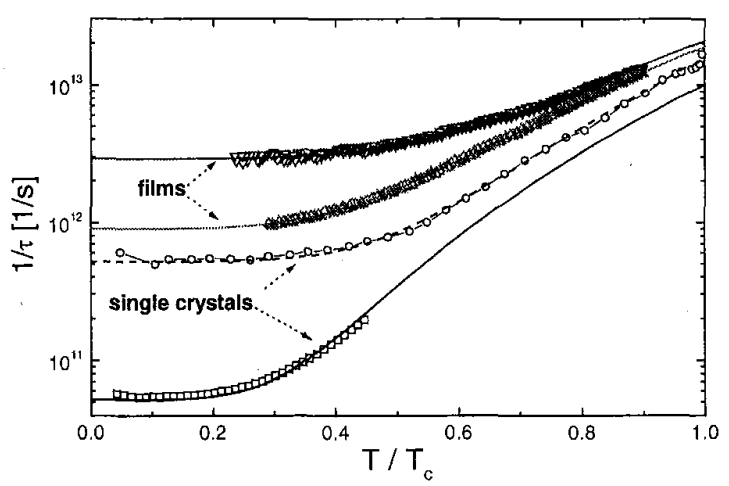

Fig. 3. Experimentally determined scattering rates of the thermally excited quasiparticles in YBCO single crystals [4] and our YBCO films with different defect densities. The lines represent fits to the experimental data according to eq. (3). 


\section{Microwave Power Handling}

For most applications the microwave power handing capability of the high- $\mathrm{T}_{\mathrm{c}}$ (HTS) films is even more important than the reduction of $R_{s}$. In ref. [6] we demonstrated that our films with high defect densities can sustain microwave field amplitudes which belong to the highest reported so far for unpatterned single-sided YBCO films. Although the temperature dependence of the maximum $\mathrm{rf}$ field levels follow the empirical expression $B_{\mathrm{HF}(\max )} \propto\left[1-\left(T / T_{\mathrm{c}}\right)^{2}\right]$, which is often observed in HTS films [7], it cannot unambiguously be related to the nonlinear mechanism, which could be of thermal and/or magnetic origin [8]. Whereas local heating at dissipative defects presents the most probable field limitation in typical HTS films below about $60 \mathrm{~K}$, flux penetration at the lower critical field $B_{\mathrm{cl}}$ is expected to dominate the nonlinear behavior as the temperature approaches $T_{\mathrm{c}}$.

Therefore, in order to check which mechanism is responsible for the nonlinear behavior in HTS films, we introduced artificial defects in form of patterned micrometer holes (so called antidots) which have successfully been used to improve the critical properties of YBCO films [9] and the sensitivity of HTS active devices [10] by means of flux pinning. In the first place we analyzed the impact of the antidots upon the rf properties at low power. Then coplanar stripline resonators were partially equipped with antidots and the resulting power handling was characterized as a function of an applied dc induction, $\mathrm{B}_{\mathrm{dc}}$.

\section{A. $R_{\mathrm{s}}$ of YBCO films with antidots}

Fig. 4 show a typical antidot lattice which is patterned via optical lithography and ion beam etching into a YBCO film on sapphire. A typical diameter $d=1.5 \mu \mathrm{m}$ of the antidots and lattice parameter of $10 \mu \mathrm{m}$ have been used for our experiments [11]. Fig. 5 presents the temperature dependence of $R_{S}$ of a YBCO film before and after the patterning of an antidot lattice measured at low rf power in a dielectric resonator. The antidot lattice causes a slight increase of the surface resistance. It will be discussed the framework of different models [11]. The resulting theoretical predictions of these models are given in Fig. 6.

1) Summation of $R_{s}$ contributions

In the simplest model the surface resistance can be assumed to be given by combination of the contribution of the YBCO matrix and the antidots. This would be valid if the rf field would penetrate through the antidots into the substrate.

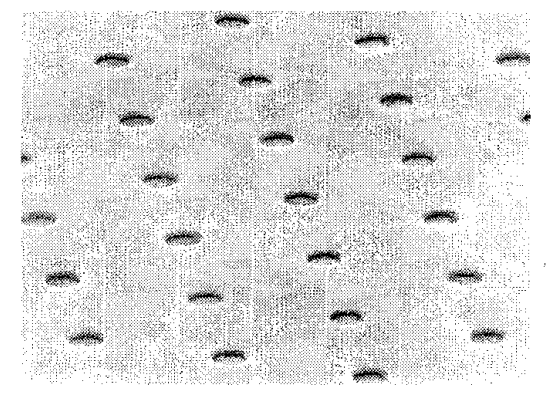

Fig. 4. YBCO film with a hexagonal antidot lattice with periodicity of 10 $\mu \mathrm{m}$ and antidot diameter of $1.5 \mu \mathrm{m}$.

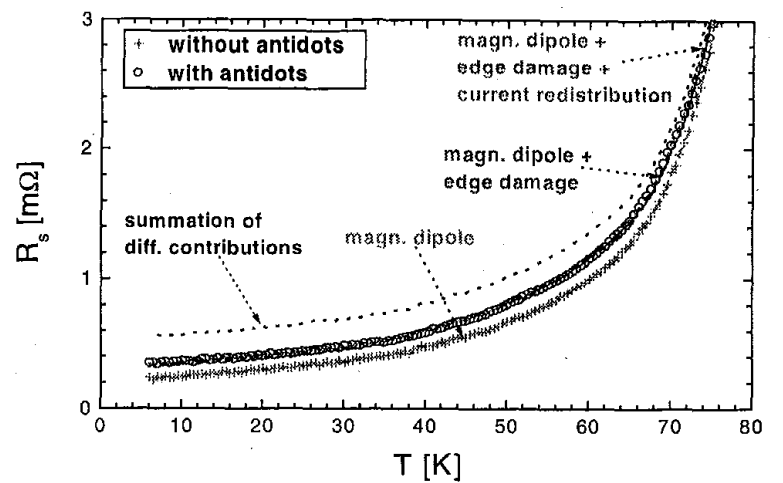

Fig. 5. Comparison of the temperature dependence of a YBCO film without and with antidots measured in a dielectric resonator at $19 \mathrm{GHz}$. Additionally the theoretical predictions of different models are given.

Since the losses in the sapphire substrate are negligible $\left(\tan \delta \approx 10^{-6}-10^{-8}\right.$ for $77 \mathrm{~K}$ and $\mathrm{GHz}$ ), the losses at the $\mathrm{Cu}$ housing of the dielectric resonator have to be considered, thus:

$$
R_{s}=\alpha R_{s, C u}+(1-\alpha) R_{s, Y B C O} \quad,
$$

with $\alpha=0.03$ denoting the geometrical factor describing the fraction of the surface occupied by antidots. Inserting experimental values for the surface resistance of $\mathrm{YBCO}$ and $\mathrm{Cu}$ yield far to large values for $\mathrm{R}_{s}$ over the whole temperature range (see dashed line in fig. 5).

\section{2) Magnetic dipole}

In this model the antidots are considered as sources for magnetic dipole radiation which describes the modification of the rf field by antidots. This estimation is reasonable since the antidots with diameter $d \approx 1.5 \mu \mathrm{m}$ are small compared to the rf wavelength $\lambda_{\mathrm{HF}}(19 \mathrm{GHz}) \approx 15 \mathrm{~cm}$. The additional loss can be characterised by the quality factor

$$
1 / Q_{s}=P_{\text {rad }} /(\omega W)
$$

which describes the scattering process of the dipole. Here $W$ denotes the field energy stored in the resonator and $P_{\text {ral }}$ the dipole-radiated power which is given by [12]:

$$
P_{\text {rad }}=(8 N / 27 \pi)(d / 2)^{6}(\omega / c)^{4} Z_{o} \cdot|\vec{H}|^{2}
$$

where $\mathrm{N}$ denotes the number of antidots, $\mathrm{c}$ the speed of light, $Z_{0} \approx 377 \Omega$ the wave impedance in vacuum and $H$ the magnetic field amplitude at the dipoles. The stored field energy $W=W_{\text {magn }}+W_{e l}$ is estimated by [13]:

$$
W=1 / 2 \cdot\left(\int \vec{B} \cdot \vec{H} d V+\int \vec{D} \cdot \vec{E} d V\right) \approx \mu_{o} V \cdot|\vec{H}|^{2}
$$

In first approximation the magnetic field amplitude at the dipoles is similar to that in the sapphire cylinder. Therefore the scattering quality factor can be calculated according to

$$
Q_{s}=216 \pi \omega \mu_{0} c^{4} V /\left(N d^{6} \omega^{4} Z_{o}\right)
$$

$\mathrm{V}$ denotes the volume of the sapphire cylinder, in which the rf field in the dielectric resonator is concentrated. The dimensions of the sapphire cylinder are $\mathrm{r}=\mathrm{h}=2.9 \mathrm{~mm}$ ( $\mathrm{r}$ : radius, $h$ : height), therefore $V \approx 8 \cdot 10^{-8} \mathrm{~m}^{3}$. The base of the sapphire cylinder and thus the measured area of the YBCO film is about $26.4 \mathrm{~mm}^{2}$. With the geometry of the antidot lattice one obtains $N \approx 2.6 \cdot 10^{5}$. Thus, the scattering quality factor $Q_{s}$ is 
given by an extremely high value of $\mathrm{Q}_{\mathrm{s}} \approx 5 \cdot 10^{16}>>\mathrm{Q}_{\mathrm{YBCO}}$. The quality factor of $Y B C O$ thin films in the dielectric resonator is limited at low temperatures by $\mathrm{Q}_{Y B C O}<120000$. Therefore, the scattering losses of the rf field in antidots can be neglected and can not explain the experimental results (see fig. 5).

3). Magnetic dipole plus damaged antidot edge

This model is in principle a combination of the two previous models. The antidots are considered to act as magnetic dipole, but additional contributions are obtained from a damaged, normal conducting YBCO region at the edge of the antidots. Thus, the resulting surface resistance consists of the three contributions:

$$
R_{s}=\alpha R_{s, A D}+\beta R_{s, Y R C O, N L}+(1-\alpha-\beta) R_{s, Y B C O, S L},
$$

$\alpha$ and $\beta$ denote the surface fraction of the antidots and the normal conducting $\mathrm{YBCO}$ regions, respectively. Inserting experimental values for the different contributions yields a good agreement between the experimental data and the theory for $\beta=0.0013$. This corresponds to a $\sim 20 \mathrm{~nm}$ broad damaged area around each antidot. This result seems reasonable since for instance ion-beam etched submicrometer bridges revealed damaged regions of $20-30 \mathrm{~nm}$ width [14]. Further considerations of effects of current redistribution (see fig.5) [11] only yield a slight improvement of the agreement and will not be discussed here.

\section{B. Power handling capability of YBCO films with antidots}

Finally, first tests of the power handling capability have been performed. For this purpose coplanar stripline resonators have been patterned into YBCO films on sapphire and $\mathrm{LaAlO}_{3}$. The resonance frequency was $\mathrm{f}_{0}=1.4 \mathrm{GHz}$. One of the resonators is provided with an antidot lattice $(\mathrm{d}=1.5 \mu \mathrm{m}$, periodicity $10 \mu \mathrm{m}$ ) in the central part of the stripline. The resulting dc magnetic field dependence of the resonators indicates a potential improvement obtained by antidots. Fig. 6 presents the normalised values of the maximum circulating rf power at $f_{0}$ as function of the applied dc field. The resonator with antidots shows a much weaker field dependence, i.e. $P_{\max } \propto B^{1 / 2}$ in contrast to $P_{\max } \propto B$ for the resonator without antidots. This difference in field dependence has to be ascribed to the mobility of vortices which is modified by the presence of antidots.

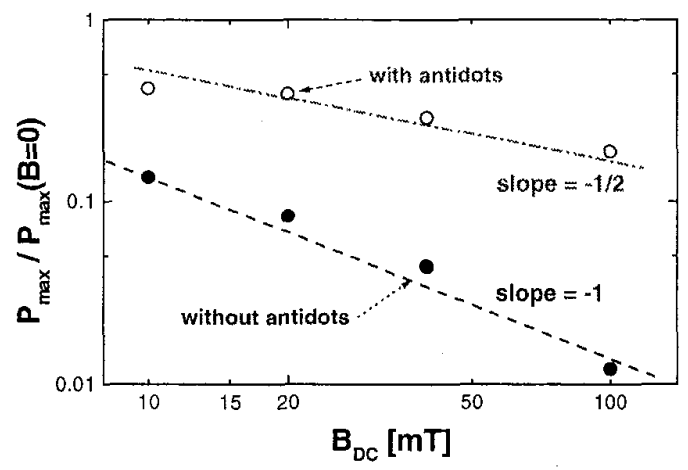

Fig. 6: Magnetic field dependence of the normalised circulating power up to which linear behaviour is observed in coplanar YBCO stripline resonators with and without antidots measured at $60 \mathrm{~K}$.

\section{CONCLUSION}

In this paper, we demonstrate the potential of defects for optimizing the microwave properties of HTS thin films. First, microscopic $\mathrm{Y}_{2} \mathrm{O}_{3}$ precipitates, which can be created in YBCO thin films by modification of the deposition process, serve as ideal scattering centres for quasiparticles and thus, lead to a considerable reduction of the microwave surface resistance. Second, the modification of $R_{s}(T)$ can be explained in terms of the two-fluid model and yields values for scattering rates. Third, the impact of artificial defects, antidots, on the microwave properties are analyzed. $R_{s}$ measurements demonstrate that the ion beam etching creates a $\sim 20 \mathrm{~nm}$ broad damaged area at the edge of the antidots. This proved to be an ideal test for etching processes. Finally, first results on the power handling capability of YBCO thin film resonators indicate that the magnetic contribution to the nonlinear behavior can be reduced by antidots. The implementation of antidots has been proven to be an ideal and easy tool to improve HTS thin film devices [10]. It might be of use for microwave applications as well. This will be analysed in the future.

\section{ACKNOWLEDGMENT}

The authors like to acknowledge A.G. Zaitsev, A.I. Braginski, N. Klein, U. Krüger, M.A. Hein, O. Vendik, R. Kutzner, H.P. Bochem, and R. Ott.

\section{REFERENCES}

[1] J. Einfeld, P. Lahl, R. Kutzner and R. Wördenweber, G. Kästner Reduction of the microwave surface resistance in YBCO thin films by microscopic defects, accepted for publication in Physica $\mathrm{C}$

[2] M. A. Hein, A. Cassinese, S. Hensen, T. Kaiser, G. Müller, M. Perpeet, J. Supercond., vol 12 (1999) 129

[3] S. Orbach-Werbig, PhD thesis, University of Wuppertal, WUB-DIS 94-4 (1994)

[4] D. A. Bonn, R. Liang, T. M. Riseman, D. J. Baar, D. C. Morgan, K, Zhang, P. Dosanjh, T. L. Duty, A. MacFarlane, G. D. Morris, J. H. Brewer, W. N. Hardy, C. Kallin, A. J. Berlinsky, Phys. Rev. B 47 (1993) 11314; A. Hosseini, R. Harris, S. Kamal, P. Dosanjh, J. Preston, R. Liang, W. N. Hardy, D. A. Bonn, Phys. Rev. B60 (1999) 1349

[5] see e.g. J. R. Waldram in Superconductivity of Metals and Cuprates, IOP Publ. Ltd 19956, Bristol and Philadelphia, p. $230-266$

[6] A. G. Zaitsev, R. Kutzner, R. Wördenweber, T. Kaiser, M. A. Hein, G. Müller, J. Supercond. 11 (1998) 361

[7] M. A. Hein, Supercond. Sci. Technol. 10, 867 (1997)

[8] M. A. Hein, C. Bauer, W. Diete, S. Hensen, T. Kaiser, G. Müller, and H. Piel, J. Supercond. 10, 109 (1997)

[9] A. Castellanos, R. Wördenweber, G. Ockenfuß, A. v. d. Hart, K. Keck, Appl. Phys. Lett. 71 (1997) 962

[10] P. Selders, A. Castellanos, M. Vaupel, R. Wördenweber, IEEE Trans. Appl. Supercond. 9 (1999) 2967; P. Selders und R. Wördenweber, Appl. Phys. Lett., vol. 76, pp. 3277, 2000.

[11] J. Einfeld, Bericht d. Forschungszentrum Jülich, Jül-3773, ISSN 09442952, D38 (2000)

[12] J. A. Stration, Electromagnetic Theory, McGraw-Hill, New York (1941)

[13] J. D. Jackson, Classical Electrodynamics, Walter de Gruyter, Berlin (1983)

[14] J. Schneider, H. Kohlstedt, R. Wördenweber, Appl. Phys. Lett. $\underline{63}$ (1993), 2426. 